\title{
Strain-based Electrical Properties of Systems of Carbon Nanotubes Embedded in Parylene
}

\author{
David D. Allred \\ allred@byu.edu \\ Jon Brame \\ Johnathan Goodsell \\ Stephanie Getty
}

Follow this and additional works at: https://scholarsarchive.byu.edu/facpub

Part of the Astrophysics and Astronomy Commons, and the Physics Commons

\section{Original Publication Citation}

Jon Brame, Stephanie Getty, Johnathan Goodsell, and David Dean Allred, "Strain-based Electrical Properties of Systems of Carbon Nanotubes Embedded in Parylene," in Nanowires and Carbon Nanotubes - Science and Applications, edited by P. Bandaru, M. Endo, I.A. Kinloch, A.M. Rao (Mater. Res. Soc. Symp. Proc. 963E, Warrendale, PA, 27)

\section{BYU ScholarsArchive Citation}

Allred, David D.; Brame, Jon; Goodsell, Johnathan; and Getty, Stephanie, "Strain-based Electrical Properties of Systems of Carbon Nanotubes Embedded in Parylene" (2007). Faculty Publications. 951.

https://scholarsarchive.byu.edu/facpub/951 


\title{
Strain-based Electrical Properties of Systems of Carbon Nanotubes Embedded in Parylene
}

Jon Brame ${ }^{1}$, Stephanie Getty ${ }^{2}$, Johnathan Goodsell ${ }^{1}$, and David Dean Allred ${ }^{3}$

${ }^{1}$ Physics and Astronomy, Brigham Young University, Provo, UT, 84602

${ }^{2}$ Code 541, NASA GSFC, Greenbelt, MD, 20771

${ }^{3}$ Physics and Astronomy, Brigham Young University, N265 ESC, Provo, UT, 84602

\begin{abstract}
We have fabricated flexible electronic devices to test the strain-based change in resistance of a network of single-walled carbon nanotubes (SWCNTs) for use in microscale, high resolution magnetometry. To do this, we first develop a simple, reliable method to obtain catalyst nanoparticles for carbon nanotube growth through indirect, thin-film evaporation. Next we fabricate a two-terminal SWCNT device on a rigid substrate. We then transfer the device, intact, to a flexible substrate for strain testing. Herein, we report progress in growth and measurement techniques.
\end{abstract}

\section{INTRODUCTION}

Single-walled carbon nanotubes (SWCNTs) have been the subject of intensive research efforts in recent years, owing to their remarkable electronic and mechanical properties. It is not surprising, perhaps, that SWCNTs display a large strain-induced response in electronic resistance, suggesting their application in strain sensing devices [1-6]. Our approach to developing SWCNTs into a modular, conformal strain sensor is to employ parylene as a flexible substrate. Toward this goal, we have achieved dense growth of a network of SWCNTs, established electrical contact to the network, and finally, demonstrated transfer of an intact SWCNT device to a flexible parylene substrate. Preliminary strain measurements have been performed, and the results are discussed below.

\section{EXPERIMENT}

\section{Thin film catalyst and Growth}

A catalyst-assisted chemical vapor deposition process is used to grow SWCNTs on a silicon substrate, capped with $500 \mathrm{~nm}$-thick oxide. Iron nitrate catalyzed single walled carbon nanotube growth is well documented in the literature [7-8], and more recently, SWCNT growth using vacuum-deposited thin film iron has also been reported [7-10]. Both methods of catalyst deposition were explored in this work, and superior results have been achieved with a simple, reliable method for depositing thin film iron catalyst in high vacuum.

The iron catalyst was deposited using an indirect evaporation at approximately $10^{-6} \mathrm{Torr}$ in a high vacuum chamber [11]. Contrary to traditional thin film evaporations, the substrate was oriented facing away from the iron source. Iron was then evaporated at a rate of $0.5 \AA / s$ to a 
direct thickness of $20 \AA$ according to the thickness monitor. This indirect evaporation yields discrete nanoparticles of iron on the silicon dioxide surface.
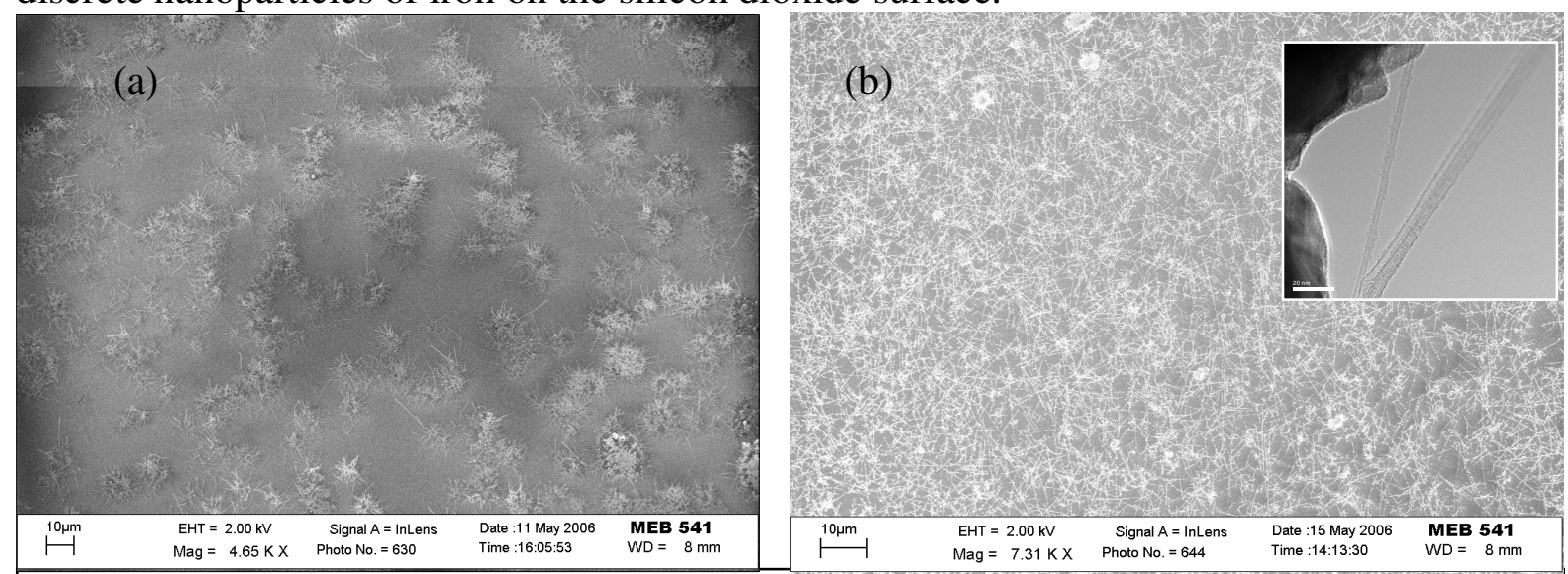

Figure 1: (a) SEM image of carbon nanotubes (CNTs) grown with Iron Nitrate catalyst. (b) CNTs grown with thin film iron catalyst. The inset (what inset?) is a TEM image of the thin film iron growth indicating the presence of SWCNT and nanotube bundles resulting from the thin film growth process. The thin film catalyst consistently yielded much denser growth, and lower incidence of catalyst agglomeration.

The substrate was then placed in a growth furnace and heated to $950^{\circ} \mathrm{C}$ for chemical vapor deposition (CVD) carbon nanotube growth. During growth, methane, ethylene, hydrogen and humid argon were flowed over the substrate for 5 minutes. See figure 2 for complete fabrication schematic. The resulting SWCNT network was then imaged in a scanning electron microscope (SEM), equipped with an in-lens detector, for qualitative analysis. Energy-dispersive x-ray spectroscopy (EDX) was used for elemental characterization, indicating the presence of carbon, iron, oxygen, and silicon.

Both types of catalyst, solution-cast iron nitrate and thin film iron, were explored in this work, and a comparison of growth results for each is shown in figure 1. Part (a) shows SWCNT growth using iron nitrate catalyst, deposited from solution. A high density of agglomerated catalyst can be seen, with short SWCNTs growing from each discrete catalyst island. In contrast, figure 1(b) shows a transmission electron micrograph (TEM) image of a SWCNT network grown using thin film Fe catalyst. A high-density network of long, interconnected SWCNTs can be seen, with a low incidence of large catalyst islands.

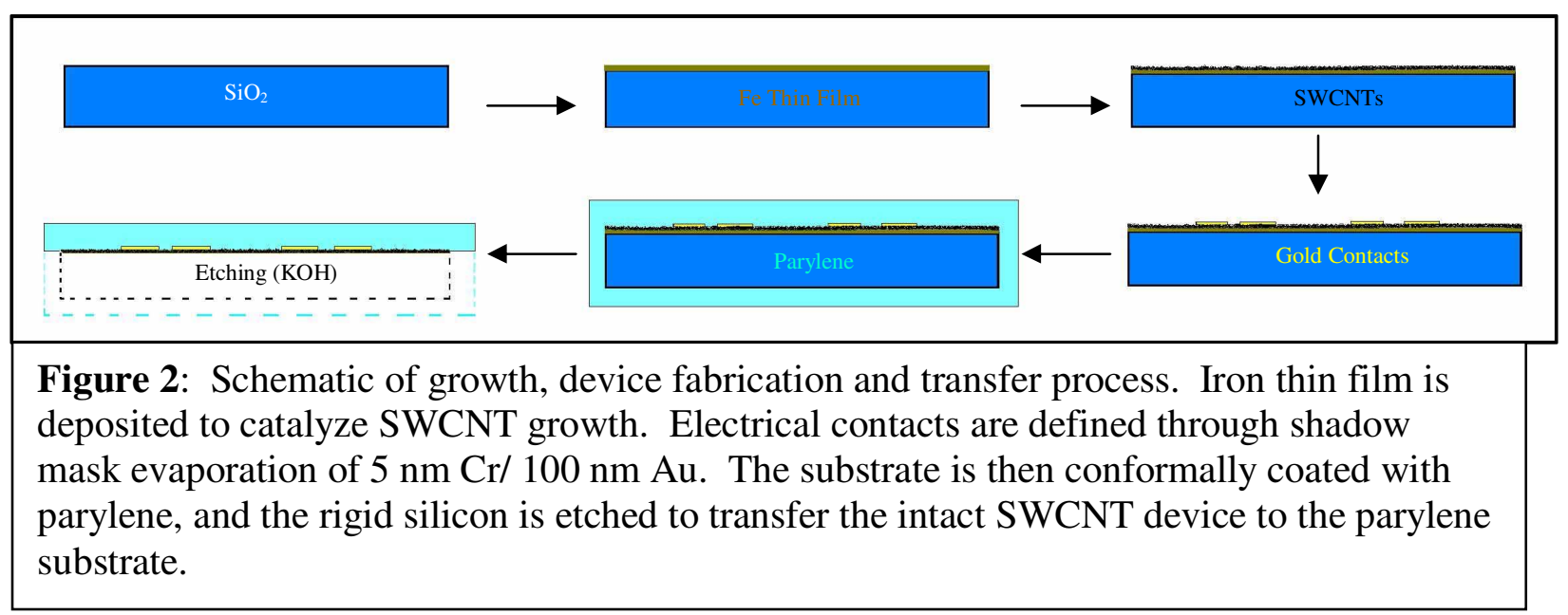




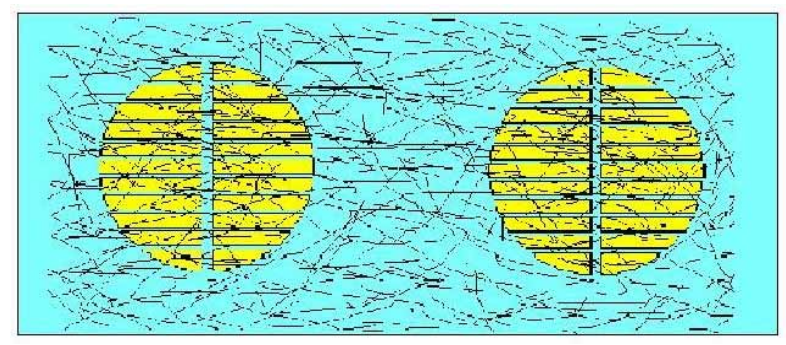

Figure 3: Schematic of the electrode pattern, bridged by a network of SWCNTs.

\section{Device Evaporation}

To fabricate two-terminal SWCNT network devices, we deposited a thin-film layer of chromium $(5 \mathrm{~nm})$, followed by a layer of gold $(100 \mathrm{~nm})$ on top of the SWCNT network, using a shadow mask to define a series of highly redundant contacts (see figure 3 ). The spacing between contacts, or the device length, is 50 microns. Electrical characterization of each device was preformed using a probe station. Two-terminal current-voltage data were obtained by applying a dc voltage (Sorensen XT15-4 power supply) and measuring current using a Stanford Research Systems SR570 low-noise current pre-amplifier. Data were acquired using LabView.

\section{Device Transfer}

Once the devices were characterized, the next step was to transfer them to a flexible substrate. Parylene-C (p-xylene) was chosen as the material for the flexible substrate because of its excellent chemical resistance and its ability to coat samples with high levels of uniformity and penetration. Other groups mention coating nanotubes with parylene-c for the purpose of nanotube protection, but have not attempted a transfer onto a parylene substrate [13-14]. We placed the device to be coated inside a PDS 2010 Parylene coater and deposited 19 grams of parylene throughout the chamber, resulting in an actual coating thickness of $25 \mu \mathrm{m}$.

Using standard microfabrication techniques, release of the SWCNT-parylene system was accomplished by wet etching the silicon substrate. Since the parylene coating process results in a conformal encapsulation of the substrate, an oxygen etch was used to remove the back side of the parylene, exposing the back side of the oxidized silicon substrate. The oxide was then removed using a dry etch process to reveal the bulk silicon substrate. Finally, the silicon was removed
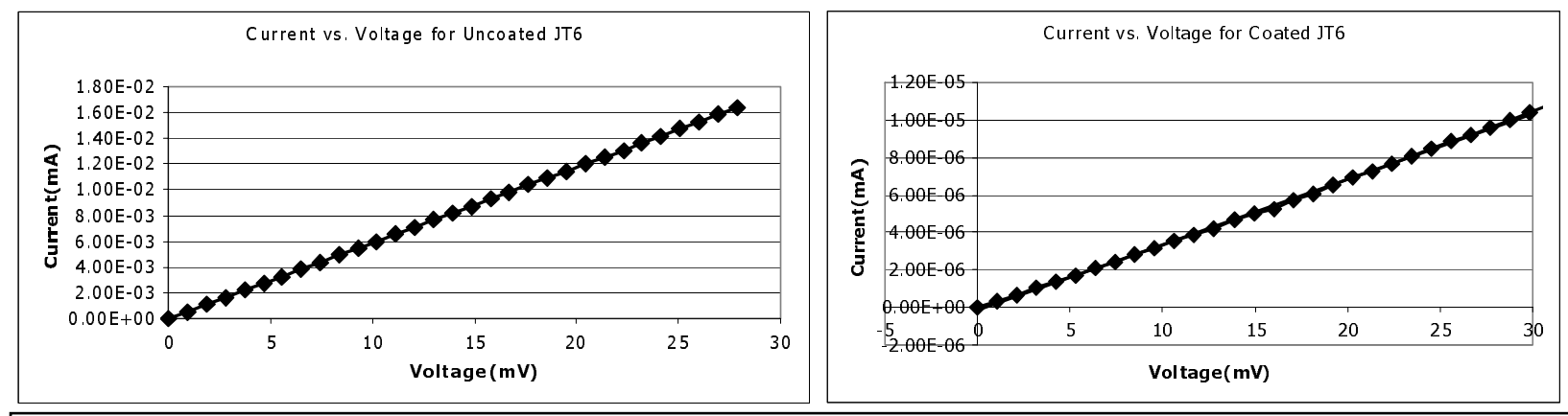

Figure 4: (a) IV curve for SWCNT device before parylene coating. (b) IV curve for same device after parylene coating. The electrical resistance is unchanged by the transfer indicating the device transfer was successful. 
using a wet etch process, and the pre-formed SWCNT devices remained intact, partially embedded in the remaining parylene.

Figure 5 shows a SEM image of the nanotube network between two gold contacts after successful transfer. Testing of these devices after the transfer showed that the electrical integrity of the device is maintained. Figure 4 shows IV curves for one device before and after transfer. Preliminary strain tests indicate that the SWCNTs are able to withstand bending and/or stretching to investigate electromechanical properties of the structure.

\section{$\underline{\text { Device Testing }}$}

To test the strain-based resistance change of the SWCNT network, we adapted our probe station so that we could simultaneously stretch the parylene device

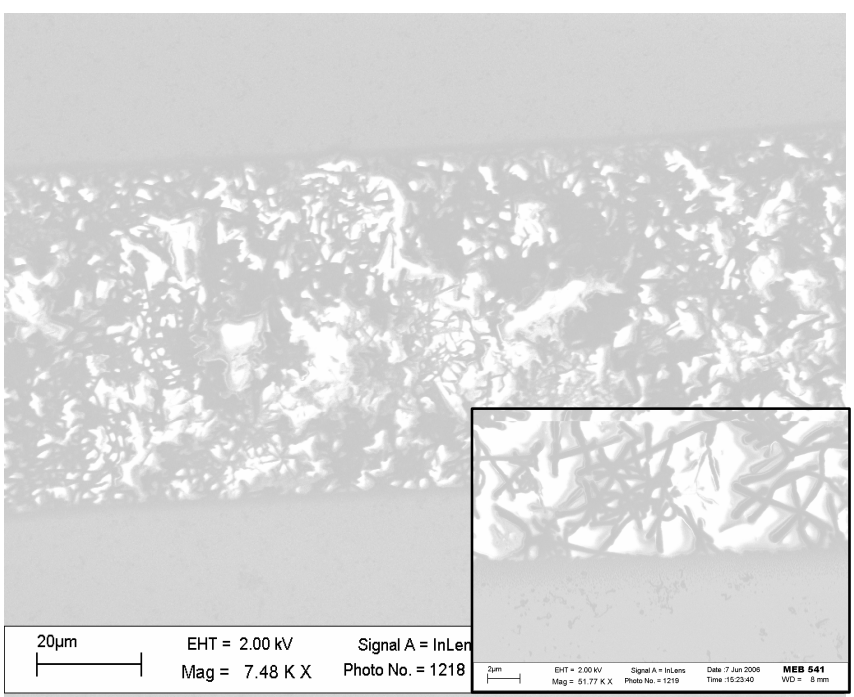

Figure 5: SEM image of SWCNT network embedded in parylene between two gold contacts. Inset shows higher magnification of the interface between the SWCNTs and the gold contact. while measuring the resistance of the individual contacts. A micromanipulator (Signatone) was used to stretch the parylene-bound devices along the device length, and then used the two-point probe station to monitor the current-voltage characteristics of the device under study. In order to reduce contact effects, we mechanically attached opposing ends of the substrate onto a separate chip carrier (see fig 2) and wire bonded to the device. Tungsten probes were then contacted to the rigid chip carrier, using indium pellets to absorb any vibration. However, even with these precautions, the limiting factor on our measurement precision was the issue of contact resistance.

We measured the change in electrical resistance as a function of elongation for each successful SWCNT network device. Reversibility of the process is critical to demonstrating that any resistance change is due to strain, rather than contact effects. To address this issue, a

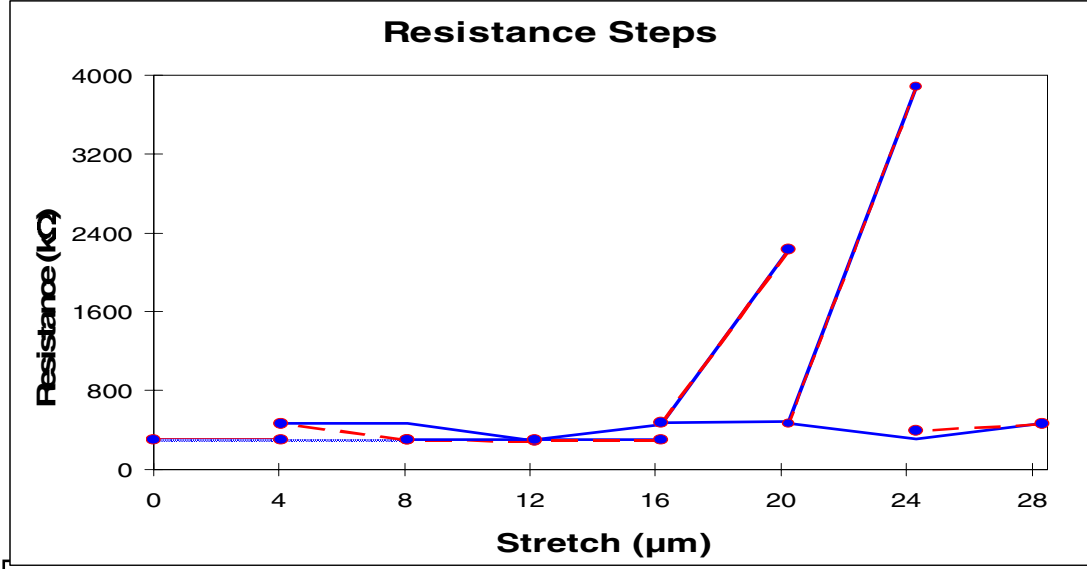

Figure 6: The slope of the lines between $4 \mu \mathrm{m}$ stretch sets indicates that the resistance increases reversibly with sawtooth pattern in the stretching of the substrate was used. For each position measurement, we first applied an elongation to the sample of one-eighth $(1 / 8)$ of a turn on the fine manipulator $(4 \mu \mathrm{m})$ and measured the device. We then retracted the sample by $1 / 8$ turn, measured the device, and finally elongated the sample by two more $1 / 8$ turn steps (total 8 $\mu \mathrm{m})$ measuring at the intermediate steps. 


\section{DISCUSSION}

\section{Strain-resistance testing}

Preliminary results of strain testing show indications of reversible, strain-based resistance increase (piezoresistance) in the SWCNT network. Figure 6 shows two-terminal resistance in a SWCNT network device as a function of micromanipulator position, relative to the starting position. We must emphasize that these results are preliminary, the error in the data is large, and no conclusive evidence is seen for a piezoresistive response in the device under study. However, we offer an interpretation of the data that is consistent with experimental observations.

In the data curves are shown in figure 6 the experiment begins on the left with no extension and ends on the right with the dashed curve ending at 24 microns. The solid segments represent the resistance elongation, and the dashed segments represent resistance after retraction. Where the curves overlap, the dashed lines cannot be seen. As shown in the first three points on the left of the graph, the device was initially stretched by two steps of $4 \mu \mathrm{m}$ and then relaxed in one step by $4 \mu \mathrm{m}$ to monitor for hysteresis, or evidence that the signal is due to mechanisms other than piezoresistance. We speculate that the device is in slack for the low stretch values, due to the scatter in the data and the hysteresis seen between 4 and $12 \mu \mathrm{m}$ stretch.

We observe that all hysteretic events are characterized by a small resistance change of approximately $200 \mathrm{k} \Omega$. Furthermore, the resistance appears to be oscillating between discrete values of $\sim 400$ and $\sim 600 \mathrm{k} \Omega$. These two levels of resistance may suggest that the conduction takes place along a single channel that is poorly contacted to the network.

Only two stretch points are significantly different from the others: $20 \mu \mathrm{m}$ and at $24 \mu \mathrm{m}$. Most notably, these two large resistance increases are reversible and consistent with the large piezoresistance that we expect with an increase in strain. We speculate that the two increases actually represent a single piezoresistive signature, but that lack of precision in the micromanipulator may have produced a source of error in our stretch measurement. From these limited data, it is not possible to draw quantitative conclusions about the performance of our conformal strain sensor, and it is clear that more careful testing is required. Continuing efforts are underway to enable a more accurate measurement method.

\section{CONCLUSION}

We have demonstrated a method for transferring intact SWCNT electrical devices to a flexible parylene substrate. We have also demonstrated a simple, reliable method, through indirect thin-film evaporation, to obtain catalyst nanoparticles for carbon nanotube growth. The piezoresistive properties of our resultant flexible SWCNT devices have been investigated, though conclusive, quantitative strain-dependent resistance modulation has not been shown. Preliminary results indicate, however, that the parylene transfer method shown here is promising for developing a robust, modular strain sensor using a SWCNT network. 


\section{ACKNOWLEDGEMENTS}

The authors would like to thank Dr. Richard Vanfleet of Brigham Young University, Provo, UT, for his support in TEM imaging. Research was done at NASA Goddard Space Flight Center supported by GSFC Faculty Student Team Program (FSTP) (Exploration Systems Mission Directorate), Rocky Mountain Space Grant Consortium and the GFSS Directors Discretionary Fund.

\section{REFERENCES}

1. T. Tombler, C Zhou, L Alexseyev, J Kong, H Dai, "Reversible electromechanical characteristics of carbon nanotubes under local-probe manipulation," Nature vol. 405, p. 769 (2000).

2. A. Maiti, A. Svizhenko, and M. P. Anantram, "Electronic Transport through Carbon Nanotubes: Effects of Structural Deformation and Tube Chirality," Phys. Rev. Lett. vol. 88, p. 126805 (2002).

3. J. Cao, Q. Wang, and H. Dai, "Electromechanical Properties of Metallic, Quasimetallic, and Semiconducting Carbon Nanotubes under Stretching," Phys. Rev. Lett. vol. 90, p. 157601 (2003).

4. C. Stampfer, A Jungen, C Hierold - Sensors, "Fabrication of Single-Walled CarbonNanotube-Based Pressure Sensors," Nano Letters vol. 6, p. 233 (2006).

5. Y. Su, A. G. R. Evans, and A. Brunnschweiler, "Micromachined silicon cantilever paddles with piezoresistive readout for flow sensing," J. Micromech. Microeng. v. 6, p. 69 (1996).

6. P. Dharap, Z Li, S Nagarajaiah, EV Barrera, "Nanotube film based on single-wall carbon nanotubes for strain sensing," Nanotechnology vol. 15, p. 379 (2004).

7. J. H. Hafner, MJ Bronikowski, BR Azamian, P Nikolaev, " "Catalytic growth of single-wall carbon nanotubes from metal particles," Chem. Phys. Lett. vol. 296, p.195 (1998).

8. J. Kong, HT Soh, AM Cassell, CF Quate, H Dai, "Synthesis of individual single-walled carbon nanotubes on patterned silicon wafers," Nature vol. 395, p. 878 (1998).

9. R. G. Lacerda, KBK Teo, AS Teh, MH Yang, SH Dalal, "Thin-film metal catalyst for the production of multi-wall and single-wall carbon nanotubes," J. Appl. Phys. vol. 96, p. 4456 (2004).

10. K. Hata, DN Futaba, K Mizuno, T Namai, M Yumura, "Water-Assisted Highly Efficient Synthesis of Impurity-Free Single-Walled Carbon Nanotubes," Science vol. 306, p. 1362 (2004).

11. S. A. Getty, J. E. Goodsell, J. A. Brame, G. Kletetschka, and D. D. Allred, "Electronic Properties of Single-Walled Carbon Nanotubes for a Strain-Based Magnetometer," IEEE Transactions on Nanotechnology, 2006 in press.

12. P. Nikolaev, M. J. Bronikowski, R. K. Bradley, F. Rohmund, D. T. Colbert, K.A. Smith, R. E. Smalley, "Gas-phase catalytic growth of single-walled carbon nanotubes from carbon monoxide," Chem. Phys. Lett. vol. 313, p. 91 (1997).

13. R Chan, CKM Fung, WJ Li., "Rapid assembly of carbon nanotubes for nanosensing by dielectrophoretic force," Nanotechnology vol. 15, p. 672 (2004).

14. S. Miserendino, J Yoo, A Cassell, YC Tai, "Electrochemical characterization of paryleneembedded carbon nanotube nanoelectrode arrays," Nanotechnology, vol. 17, 23 (2006). 